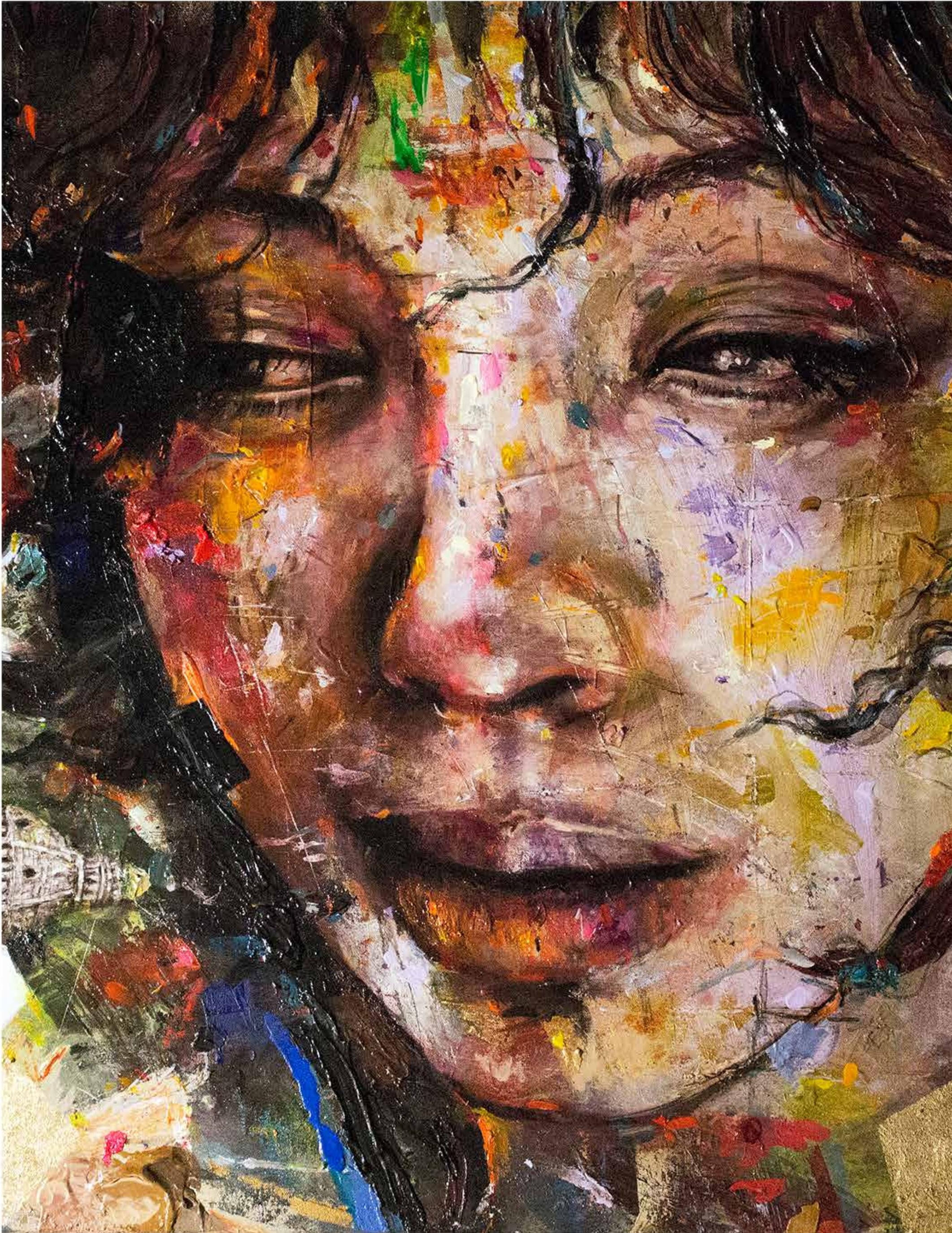




\section{Pan y rosas \\ 0.2 〜. \%}

Mientras vamos marchando, marchando a través del hermoso día Un millón de cocinas oscuras y miles de grises hilanderías Son tocados por un radiante sol que asoma repentinamente Ya que el pueblo nos oye cantar: ¡Pan y rosas! ¡Pan y rosas!

Mientras vamos marchando, marchando, luchamos también por los hombres Ya que ellos son hijos de mujeres, y los protegemos maternalmente otra vez Nuestras vidas no serán explotadas desde el nacimiento hasta la muerte Los corazones padecen hambre, al igual que los cuerpos ¡dennos pan, pero también dennos rosas!

Mientras vamos marchando, marchando, innumerables mujeres muertas

Van gritando a través de nuestro canto su antiguo reclamo de pan

Sus espíritus fatigados conocieron el pequeño arte y el amor y la belleza

¡Sí, es por el pan que peleamos, pero también peleamos por rosas!

A medida que vamos marchando, marchando, traemos con nosotras días mejores El levantamiento de las mujeres significa el levantamiento de la humanidad Ya basta del agobio del trabajo y del holgazán: diez que trabajan para que uno repose

¿Queremos compartir las glorias de la vida: pan y rosas, pan y rosas!

Nuestras vidas no serán explotadas desde el nacimiento hasta la muerte Los corazones padecen hambre, al igual que los cuerpos

ipan y rosas, pan y rosas!

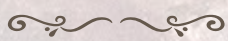

\section{James Oppenheim}




\title{
3. La segregación laboral vertical y horizontal. Elementos estructurales del mercado laboral hondureño: 2001, 2005 y 2010
}

\author{
Martha Lilian Cálix \\ DOI: 10.5377/pdac.v14i0.6851 \\ Recibido: 29/04/2018 Aceptado: 01/07/2018
}

Resumen: La segregación laboral vertical y horizontal es una problemática internacional que atañe a todos los países con mayor o menor intensidad. En el caso de Honduras no es un hecho cíclico, mucho menos estacional pues esta irregularidad se ha sostenido a lo largo de los años; como lo evidencian los recuentos de la Encuesta Permanente de Hogares de Propósitos Múltiples (EPHPM) realizada por el Instituto Nacional de Estadísticas (INE) de los años 2001, 2005, 2010. Los desajustes en la distribución de las mujeres y los hombres en las ocupaciones laborales pueden verificarse por un índice de segregación. Este método ampliamente empleado en investigaciones fue creado en 1955 por Duncan y Duncan. Con base en los datos censales, o encuestas de hogares, el método concede la posibilidad de verificar la clasificación de la fuerza laboral, asimismo demostrar el grado en el que difiere la participación relativa de los hombres y las mujeres. Indicadores como ocupación principal de la PEA, reveló que el sector primario, en el ámbito rural, sostiene una alta concentración de mano de obra masculina, lo cual remarca el índice de disimilitud laboral. Para el período de estudio alcanzó valores porcentuales entre $54.8 \%$ y $64.7 \%$. Con relación a los ingresos monetarios, las cifras demostraron que en las grandes ciudades (Tegucigalpa-Comayagüela y San Pedro Sula), en promedio, los hombres son mejor retribuidos que el resto de los grupos en los dominios complementarios. La aplicación del índice de Duncan dejó ver la cuantificación del fenómeno de la segregación laboral, así como el estrecho vínculo, del mismo, con los tradicionales patrones de género. De igual manera la concentración de mano de obra masculina en las ocupaciones del primer sector de la economía; agricultura, ganadería y trabajos agropecuarios; la fuerza laboral de los hombres es altamente representativa en el área textil, albañilería y mecánica. La mano de obra femenina en el área rural está absolutamente excluida de las tareas de transporte.

Palabras clave: División sexual del trabajo, Índice de Duncan, ocupaciones, participación laboral.

\section{Vertical and horizontal labor segregation. Structural elements of the Honduran labor market: 2001, 2005 and 2010}

Abstract: Vertical and horizontal labor segregation is an international problem that concerns all countries with greater or less intensity. In Honduras case, it is not a cyclical event, much less seasonal, since this irregularity has been sustained over years; as evidenced by the counts of the Permanent Survey of Households of Multiple Purposes conducted by the National Statistics Institute for the years 2001, 2005, and 2010. The imbalances in the distribution of women and men in occupations can be verified by an index of segregation. This method widely used in research was created in 1955 by Duncan and Duncan. Based on census data, or household surveys, the method gives the possibility of verifying the classification of the workforce, as well as demonstrating the degree to which the relative participation of men and women differ. Indicators like the main occupation of the PEA, revealed that the primary sector, in rural areas, maintains a high concentration of male labor, which highlights the index of labor dissimilarity. For the study period, it reached percentage values between $54.8 \%$ and $64.7 \%$. In relation to monetary income, the numbers showed that in large cities (TegucigalpaComayagüela and San Pedro Sula), on average, men are better paid than the rest of the groups in the complementary domains. The application of the Duncan Index showed the quantification of the phenomenon of labor segregation, as well as its close link to traditional gender patterns. In the same way, the concentration of male labor in the occupations of the first sector of the economy; agriculture, livestock and agricultural work; the labor force of men is highly representative in the textile, masonry and mechanical areas. Female labor in rural areas is absolutely excluded from transport labor.

Keywords: Sexual division of labor, Duncan index, occupations, labor participation.

\section{Introducción}

Las diversas sociedades y su simbolismo cultural, de manera dinámica han dado significados y valoraciones al hombre y a la mujer. A raíz de esa caracterización, de la concepción de normas en el ámbito social, político, económico y cultural, diferenciadas por sexo, surge el concepto de género como categoría que permite el análisis de la diferencia que se establece entre hombres y mujeres en los diferentes contextos.

La Organización Internacional del Trabajo (OIT) en el
Informe de la 91a. reunión, 2003, en ausencia de indicadores precisos para medir de manera directa la discriminación en el trabajo utiliza los llamados indicadores supletorios válidos. Estos son: las disparidades por razón de género en las tasas de participación en la fuerza de trabajo; las tasas de desempleo; los salarios, y los trabajos en que predominan las mujeres frente a aquellos en que predominan los hombres. (OIT, 2003). Este último indicador, que marca tendencias de concentración por sexo en ocupaciones, define el concepto de segregación ocupacional por género, como la concentración desproporcionada de mujeres en cierto tipo de ocupaciones y de hombres en otras.

1 Máster en Demografía y Desarrollo. Docente de la Escuela Agrícola Panamericana “El Zamorano" e-mail: mcr_2013@yahoo.com 
Durante la última década, en la variable ocupación (a un digito) se identificó cinco ocupaciones, las cuales en términos porcentuales congregan la mayor cantidad de Población Económicamente Activa. Los hombres y las mujeres se concentran en trabajos considerados social y tradicionalmente como labores adecuadamente masculinas o femeninas; confirmando la existencia de la segregación laboral por sexo.

\section{Metodología}

El estudio se concibe como una investigación descriptiva - longitudinal, y cuantitativa. Implicó el procesamiento y análisis de datos, generados por el Instituto Nacional de Estadísticas (INE) en la década: 2001 - 2010, se hizo énfasis en los años 2001, 2005 y 2010, para hacer inferencias con relación a cambios, sus determinantes y posibles consecuencias.

La investigación se orientó a revelar la segregación ocupacional femenina en el mercado laboral de Honduras en la última década. Se tomó como unidad de análisis las mujeres insertas en el mercado laboral hondureño. Se trabajaron las categorías ocupacionales, de manera desagregada con cruce de otras variables como: sexo, ingresos, ocupación y residencia. El uso de la estadística descriptiva permitió detectar la situación laboral real desagregada por sexo.

La operatividad del Índice de Disimilitud de Duncan y Duncan, en su conjunto facilita el conocimiento del comportamiento del mercado laboral, enfocado en las ocupaciones y desagregadas por sexo. La aplicación del índice de disimilitud de Duncan y Duncan, cuyo valor real puede ser interpretado como el porcentaje de mujeres (u hombres) que tendrían que cambiar de ocupaciones para que la distribución del empleo en los dos grupos sea idénticos (Blau y Hendricks, 1979), reveló la cuantificación del fenómeno de la segregación laboral, así como el estrecho vínculo, del mismo, con los tradicionales patrones de género.

\section{Discusión de resultados}

La ocupación es sinónimo de trabajo, al realizarla como actividad principal no excluye a las personas de cumplir con una o dos jornadas laborales, adicionales, sean remuneradas o no, tal es el caso particular de las mujeres asalariadas quienes además de realizar trabajos en el ámbito público, también desempeñan trabajos en el ámbito privado o doméstico condición que se experimenta tanto en el área rural como urbana. Unido a ello es evidente la segregación ocupacional que se presenta en el mercado laboral hondureño.

\subsection{Análisis comparativo de las principales ocupaciones de la PEA según sexo y área de residencia.}

- PEA ocupada por área de residencia

Entre el período 2001- 2010 se observa un incremento de la PEA en Honduras con mayor presencia de participación para la población masculina. En general, a nivel nacional, la relación es, de cada 10 personas insertas en el mercado laboral 7 son hombres y 3 mujeres, en otras palabras, la inserción de las mujeres no se ha dado al mismo ritmo en comparación con los hombres.

- Ocupaciones de la PEA del área urbana

La dinámica de la PEA masculina y la PEA femenina urbana presentan características particulares. En el caso de la PEA femenina urbana, su ocupación en el mercado laboral en el período 2001- 2010, se concentró en actividades como comerciantes y vendedoras. Una segunda plaza laboral con alta presencia femenina la representó las ocupaciones en servicios, actividad con ascenso sostenido en el período de estudio. La tercera plaza de importancia la representó las labores como profesionales y técnicos.

En el caso de la PEA masculina en el área urbana, se destacan tres ocupaciones: en primer lugar, trabajos industriales, textil, albañilería y mecánica, en segundo lugar se desempeñan como comerciantes y vendedores; y la tercera ocupación es como profesionales y técnicos. Las tres ocupaciones en ambos casos representaron más del $50 \%$ del total de los casos (Gráfico $\mathrm{N}^{\circ} 1$ y $\mathrm{N}^{\circ} 2$ ).

\section{Gráfico № 1 Ocupaciones de la PEA feminina urbana, $2001,2005,2010$}

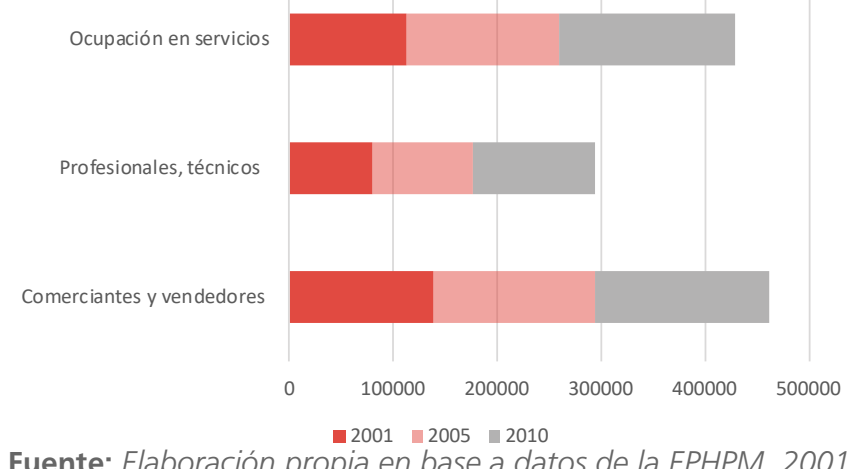

Fuente: Elaboración propia en base a datos de la EPHPM, 2001, 2005, 2010 
Gráfico № 2 Ocupaciones de la PEA masculina urbana, $2001,2005,2010$

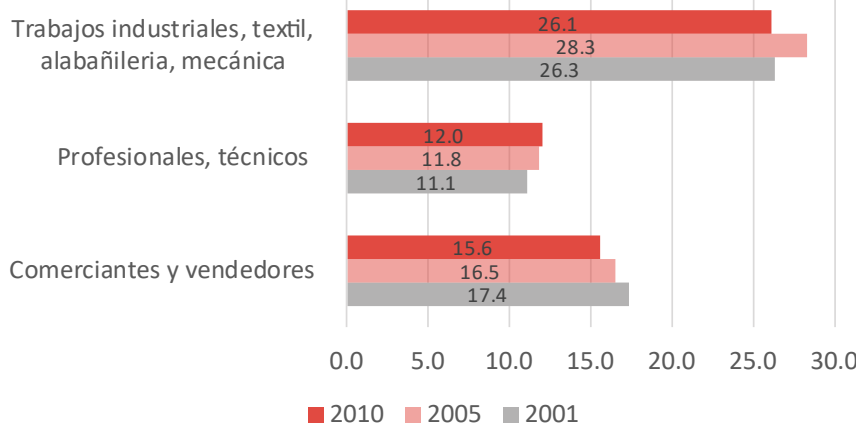

Fuente: Elaboración propia en base a datos de la EPHPM, 2001, 2005, 2010

- Ocupaciones de la PEA del área rural

En el área rural, las ocupaciones en actividades comerciales presentaron la mayor concentración de la PEA femenina. Esta actividad se realiza con mayor frecuencia desde la informalidad, complementando la tradicional ocupación de las tareas agropecuarias que ocupan el segundo lugar y una tercera posición corresponde a la ocupación en servicios (Gráfico $\mathrm{N}^{\circ} 3$ ).

\section{Gráfico $\mathbf{N}^{\circ} 3$ Ocupaciones de la PEA femenina del área} rural, 2001, 2005, 2010

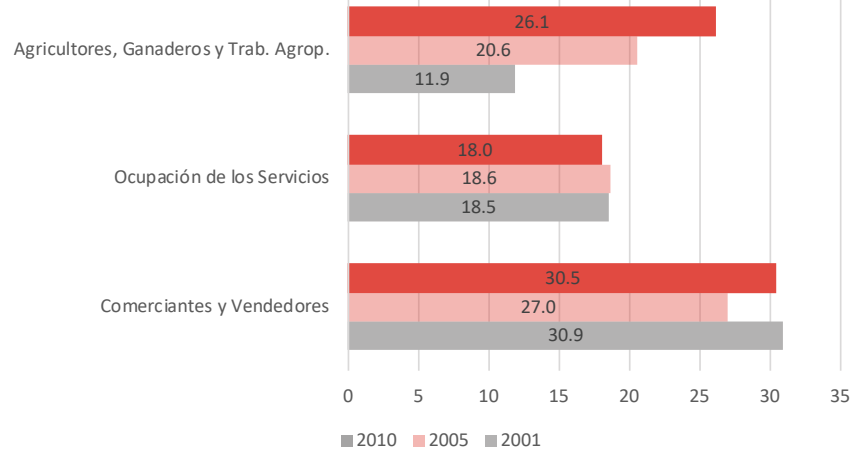

Fuente: Elaboración propia en base a datos de la EPHPM, 2001, 2005, 2010

En el caso de la PEA masculina del área rural su principal ocupación son los trabajos en la agricultura, ganadería y las actividades conexas; las mismas se convierten en las principales fuentes de trabajo e ingresos, para las familias campesinas. En los últimos años se ha incentivado los cultivos extensivos como la palma africana, melón y sandía.
El sector primario de la economía se presenta como el sector con mayor capacidad para absorber la mano de obra en el ámbito rural, considerando la tendencia de cultivos a gran escala. A las actividades agropecuarias le siguen de manera muy distante los trabajos en la industria textil, albañilería, mecánica y las actividades que se realizan en el comercio y las ventas (Gráfico $\mathrm{N}^{\circ} 3$ y $\mathrm{N}^{\circ} 4$ ).

\section{Gráfico $N^{\circ} 4$ Ocupaciones de la PEA masculina del área rural, 2001, 2005, 2010}

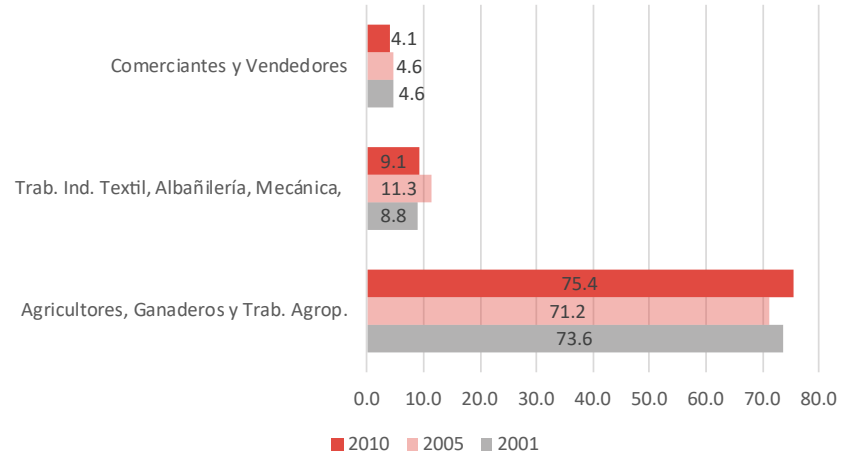

Fuente: Elaboración propia en base a datos de la EPHPM, 2001, 2005, 2010

Las ocupaciones para ambos sexos en el área rural, en el comercio y las ventas, así como las actividades en agricultura y ganadería no alcanzan la categoría de trabajo decente². Continuando el colectivo de mujeres y hombres agricultores ocupados en actividades de la economía autónoma, con claras desventajas laborales que dificultan conquistar un estado de bienestar.

\subsection{Categoría ocupacional según sexo}

Referirse a categoría ocupacional, implica reconocer la relación vertical entre la capacidad de mano de obra y los que requieren o pagan por la prestación de servicios de esa fuerza laboral. Como categorías ocupacionales se distingue, los que usan su propia fuerza, como fuente de ingresos, reconocidos como trabajadores por cuenta propia y los que venden su capacidad de trabajo en el mercado laboral, en su conjunto denominados asalariados.

La relación vertical de carácter laboral desaparece con los trabajadores por cuenta propia, ellos realizan sus actividades valiéndose de su propio trabajo personal, y usan su propia infraestructura, materiales o equipo. Los trabajadores por cuenta propia son dueños de la actividad mercantil, eventualmente complementan su ímpetu

2 Según la OIT es decente el trabajo que se realiza con respeto los principios y derechos laborales fundamentales, el trabajo que permite un ingreso justo y proporcional al esfuerzo que realiza cada trabajador, el que es incluyente, no discrimina por ninguna razón (género, raza, creencias religiosas y políticas u otros), es el trabajo que cuenta con protección social, adicionalmente respeta el y el tripartismo. 
laboral con empleados que realizan acciones específicas o adicionales a la actividad principal. El trabajo por cuenta propia suele estar identificado con el sector informal de la economía, de consecuencia, implica una nula protección social por parte del Estado.

En promedio en 2001, 2005, 2010, la categoría de cuenta propia se diferenció de las demás, categorías por la alta concentración de mujeres (94.2\%). En menor proporción $(66.7 \%)$ representó el promedio de los hombres ocupados en la misma categoría (Cuadro $\mathrm{N}^{\circ} 1$ ).

Otra categoría ocupacional es la de los trabajadores no remunerados: las personas ocupadas en una actividad productiva, dirigido por una persona que generalmente comparte vínculos consanguíneos. Los colaboradores pueden o no compartir el mismo hogar y no reciben pago en metálico por su trabajo. Al interpretar las características de la categoría de trabajadores no remunerados puede decirse que constituyen la mano de obra con mayor precariedad y vulnerabilidad laboral.

En la categoría de trabajo no remunerado los hombres, en promedio, mantienen una proporción más elevada (66\%) frente a las mujeres (34\%) (Cuadro $N^{\circ} 1$ ).

Cuadro N¹ Honduras: PEA ocupada según categoría ocupacional y sexo

\begin{tabular}{|c|l|c|c|c|}
\hline \multirow{3}{*}{ Sexo } & \multicolumn{1}{|c|}{ Categoría Ocupacional } & 2001 & 2005 & 2010 \\
\hline \multirow{4}{*}{ Hombres } & Público & 47.6 & 47.9 & 44.0 \\
\cline { 2 - 5 } & Privado & 71.2 & 74.6 & 74.8 \\
\cline { 2 - 5 } & Cta. Propia & 4.5 & 6.6 & 6.2 \\
\cline { 2 - 5 } & Doméstico & 66.3 & 61.9 & 62.1 \\
\cline { 2 - 5 } & Trabajo No Remunerado & 66.0 & 68.2 & 65.8 \\
\hline \multirow{4}{*}{ Mujeres } & Público & 52.4 & 52.1 & 56.0 \\
\cline { 2 - 5 } & Privado & 28.8 & 25.4 & 25.2 \\
\cline { 2 - 5 } & Cta. Propia & 95.5 & 93.4 & 93.8 \\
\cline { 2 - 5 } & Doméstico & 33.7 & 38.1 & 37.9 \\
\cline { 2 - 5 } & Trabajo No Remunerado & 34.0 & 31.8 & 34.2 \\
\hline
\end{tabular}

Fuente: Elaboración propia en base a datos de la EPHPM, 2001, 2005, 2010

*Datos Calculados por fila

\subsection{Categoría ocupacional según dominio desagregada por sexo}

EI INE fundamenta a través de la EPHPM las relaciones de producción, con base a las labores que las personas ocupadas realizaron su trabajo durante la semana anterior a la entrevista. Se incluye también la última ocupación de personas destituidos previa al levantamiento de la encuesta. A la característica incluyente de las y los actores de las ocupaciones, la encuesta es representativa de la realidad nacional; toma en consideración 16 de los 18 departamentos del país.

- Categoría ocupacional de los hombres según dominio

Durante los quinquenios estudiados el total de hombres asalariados mostró dos realidades paralelas: el decrecimiento en el ámbito urbano y el incremento en el espacio rural. La diferencia de puntos porcentuales entre 2001 y 2010 alcanzó $20.3 \%$ en el área urbana. Contrario al sector rural que incrementó cifras similares.

El trabajo familiar no remunerado, es una condición de labores flexibles. Se reconoce especialmente en las faenas domésticas, agrícolas estacionales, labores no estructuradas y desempeñadas a tiempo parcial o al contrario en jornadas extendidas, sin beneficios monetarios. Entre las ocupaciones de los hombres rurales, destacan los datos del trabajo familiar no remunerado, cuyas cifras en promedio, del período, superó el 76\% (Cuadro N²).

Este tipo de actividades habituales no remuneradas, tanto, como las ocupaciones por cuenta propia están lejos de alcanzar la protección social, condición que orilla a los hombres rurales a mantenerse en el círculo de la pobreza. La ocupación por cuenta propia representada por los ocupados que auto desarrollan una actividad productiva, en ausencia de empleados remunerados permanentes, eventualmente ocupan mano de obra para tareas especiales y temporales.

Con programas, proyectos o políticas públicas acertadas los asalariados de cuenta propia podrían ser los generadores de emprendimientos estables, con capacidad de calcular en sus ingresos una remuneración por su propio trabajo. Para los hombres en 2001, esta modalidad laboral, en el área rural representó el $68.1 \%$, con decrementos en los años subsiguientes, de manera particular en 2005. En el ámbito urbano, en ese mismo año, casi se duplicó las cifras (63.7\%) del 2001 y 2010 (Cuadro N²).

En 2001 y 2010 se identifica al dominio urbano como el mayor receptor de los hombres asalariados en ocupaciones domésticas (79.0 y 79.9\%). El sector privado, durante los quinquenios, en promedio (71.2\%) se revela como el mayor empleador; superando al sector público (Cuadro N²). 
Cuadro №2 Categoría ocupacional de los hombres, según dominio, 2001, 2005, 2010

\begin{tabular}{|l|c|c|c|c|c|c|}
\hline Dominio & $\begin{array}{c}\text { Total } \\
\text { Asalariados }\end{array}$ & Público & Privado & Doméstico & $\begin{array}{c}\text { Cuenta } \\
\text { Propia }\end{array}$ & $\begin{array}{c}\text { Trabajo. } \\
\text { Familiar no } \\
\text { remunerado }\end{array}$ \\
\hline \multicolumn{7}{|c|}{2001} \\
\hline Total Urbano & 59.6 & 73.5 & 57.4 & 79.0 & 31.9 & 16.3 \\
\hline Distrito Central & 21.2 & 31.4 & 19.7 & 21.4 & 9.3 & 4.3 \\
\hline San Pedro Sula & 12.2 & 5.7 & 13.0 & 23.5 & 5.7 & 2.2 \\
\hline Resto urbano & 26.2 & 36.4 & 24.6 & 34.1 & 17.0 & 9.8 \\
\hline Rural & 40.4 & 26.5 & 42.6 & 21.0 & 68.1 & 83.7 \\
\hline \multicolumn{7}{|c|}{2005} \\
\hline Total Urbano & 41.4 & 52.3 & 77.5 & 49.1 & 63.7 & 36.6 \\
\hline Distrito Central & 11.1 & 14.5 & 28.3 & 12.8 & 12.6 & 9.4 \\
\hline San Pedro Sula & 7.0 & 10.0 & 6.7 & 10.3 & 18.5 & 5.4 \\
\hline Resto urbano & 23.3 & 27.8 & 42.5 & 25.9 & 32.6 & 21.9 \\
\hline Rural & 58.6 & 47.7 & 22.5 & 50.9 & 36.3 & 63.4 \\
\hline \multicolumn{7}{|c|}{2010} \\
\hline Total Urbano & 39.3 & 51.5 & 78.8 & 79.9 & 33.6 & 17.0 \\
\hline Distrito Central & 10.6 & 15.1 & 33.5 & 9.5 & 7.9 & 4.3 \\
\hline San Pedro Sula & 6.5 & 9.5 & 6.8 & 5.9 & 4.8 & 1.6 \\
\hline Resto urbano & 22.2 & 26.9 & 38.5 & 64.4 & 20.8 & 11.1 \\
\hline Rural & 60.7 & 48.5 & 21.2 & 20.1 & 66.4 & 83.0 \\
\hline
\end{tabular}

Fuente: Elaboración propia en base a datos de la EPHPM, 2001, 2005, 2010

- Categoría ocupacional de las mujeres según dominio

Durante la etapa de estudio el colectivo femenino está muy bien incorporado en el sistema de asalariados en el ámbito urbano; en promedio representó el $74 \%$ en comparación con los varones. En cambio, en el ámbito rural, la mano de obra ocupada de las mujeres presenta un comportamiento muy inferior, entre 23 y $34.8 \%$. Se puede inferir que una de las razones es la prevalencia de las fuentes de trabajo en el sector primario. Las tareas agrícolas, ganaderas y conexas son socialmente consideradas propias de los varones.

La apertura de espacios laborales para las mujeres urbanas en la administración pública y empresa privada muestra equivalentes promedios porcentuales, los cuales superan el $76 \%$; y se concentran en el Distrito Central y el resto urbano. Por el orden de importancia de ocupación de mano de obra femenina, en el ámbito rural, los datos reflejan el trabajo familiar con porcentajes entre 43.1 y $56.2 \%$. Un comportamiento análogo refleja la ocupación de cuenta propia, seguida del trabajo doméstico (Cuadro $\mathrm{N}^{\circ} 3$ ).
La ocupación de cuenta propia, con frecuencia se refiere a tareas artesanales, o de servicios no agrícolas por ejemplo el comercio al por menor. Se argumenta que una de las tantas causas del acceso a trabajos precarios son los escasos niveles de educación. Sin embargo, se ha evidenciado que las jóvenes rurales han obtenido mayores niveles de educación que los hombres que presentan las mismas características, pero no las mismas condiciones, en este contexto se requiere profundizar sobre los fundamentos de esas restricciones laborales. 
Cuadro №3 Categoría ocupacional de las mujeres, según dominio, 2001, 2005, 2010

\begin{tabular}{|c|c|c|c|c|c|c|}
\hline Dominio & $\begin{array}{l}\text { Total } \\
\text { Asalariados }\end{array}$ & Público & Privado & Doméstico & $\begin{array}{l}\text { Cuenta } \\
\text { Propia }\end{array}$ & $\begin{array}{c}\text { Trabajo } \\
\text { Familiar no } \\
\text { remunerado }\end{array}$ \\
\hline \multicolumn{7}{|c|}{2001} \\
\hline Total Urbano & 74.5 & 71.6 & 77.5 & 68.7 & 50.9 & 45.1 \\
\hline Distrito Central & 27.5 & 29.2 & 29.0 & 20.6 & 17.9 & 15.9 \\
\hline San Pedro Sula & 14.6 & 7.5 & 18.1 & 12.4 & 8.0 & 7.1 \\
\hline Resto urbano & 32.4 & 34.8 & 30.4 & 35.6 & 25.0 & 22.1 \\
\hline Rural & 25.5 & 28.4 & 22.5 & 31.3 & 49.1 & 54.9 \\
\hline \multicolumn{7}{|c|}{2005} \\
\hline Total Urbano & 76.6 & 80.8 & 76.1 & 72.6 & 52.4 & 56.9 \\
\hline Distrito Central & 24.7 & 30.7 & 22.7 & 23.4 & 13.5 & 12.9 \\
\hline San Pedro Sula & 16.2 & 7.3 & 20.9 & 12.0 & 6.5 & 6.3 \\
\hline Resto urbano & 35.7 & 42.8 & 32.5 & 37.2 & 32.4 & 37.6 \\
\hline Rural & 23.4 & 19.2 & 23.9 & 27.4 & 47.6 & 43.1 \\
\hline \multicolumn{7}{|c|}{2010} \\
\hline Total Urbano & 75.2 & 79.3 & 75.2 & 69.7 & 46.5 & 43.8 \\
\hline Distrito Central & 25.8 & 31.8 & 23.5 & 25.9 & 12.0 & 10.2 \\
\hline San Pedro Sula & 14.6 & 7.3 & 18.7 & 10.4 & 7.3 & 7.8 \\
\hline Resto urbano & 34.8 & 40.1 & 33.1 & 33.4 & 27.2 & 25.8 \\
\hline Rural & 24.8 & 20.7 & 24.8 & 30.3 & 53.5 & 56.2 \\
\hline
\end{tabular}

Fuente: Elaboración propia en base a datos de la EPHPM, 2001, 2005, 2010

\subsection{Ingresos de hombres y mujeres según área de residencia}

Las diferencias de salarios por áreas de trabajo, especialización o espacios geográficas son parte de la dinámica del mercado laboral. Por el contrario, lo que no debe ser un componente de esa dinámica es la discriminación salarial; la cual se manifiesta cuando con iguales capacidades y condiciones laborales, las personas reciben una retribución diferenciada. Existen diversas formas de discriminación tanto laboral como salarial, las más comunes se refieren al género, edad, pertenencia a grupos étnicos y algunos casos hasta el estado conyugal.

Con base en las cifras del INE, el área de residencia no es una variable determinante para ver reflejada la paridad salarial entre hombres y mujeres. Sin embargo, ambos grupos casi han duplicado el salario entre el 2001 y 2010; los y las trabajadoras reciben mensualmente pagos superiores cuando laboran en al área urbana. Evidentemente los varones reciben remuneraciones más altas que las mujeres tanto en el ámbito urbano como en el rural (Cuadro $\mathrm{N}^{\circ} 4$ ).

El bienestar de los trabajadores y sus familias depende en gran medida del poder adquisitivo que le ofrece el salario. En este sentido, es conocido que el ingreso de los trabajadores más pobres depende básicamente del salario mínimo, el cual por ley es revisado al menos una vez al año ${ }^{3}$. De esta manera los nuevos valores salariales entran en vigor en el mes de enero del siguiente año. La decisión de incrementos es tomada de forma tripartita. Se toma en cuenta la variación del promedio de la inflación acumulada a noviembre, del año de revisión.

Con relación al promedio del ingreso laboral entre hombres y mujeres en el área urbana se mantiene la brecha salarial. Las diferencias salariales reflejan en promedio casi veinte puntos, es decir que por cada L.100.00 que devengan los varones, las mujeres solamente reciben L. 80.00. Escenario similar se presenta en el área rural, los datos del ingreso laboral en 2001 y 2010 reflejan una diferencia a favor de los hombres, (L. 1,384.90 y L. 2,903.60) superior a los 20 pp. El 2005 presenta datos atípicos, del $100 \%$ de ingreso recibido por las mujeres (L. 2000.10) el hombre (L. 1,752.10) recibió $88 \%$. Los datos revelan claramente las brechas salarias entre ambos grupos; con desventaja para las mujeres tanto en el área urbana como rural, lo cual contribuye a la discriminación salarial por sexo (Cuadro $N^{\circ} 4$ ).

3 Generalmente en el mes de diciembre. 
Cuadro N4 Relación entre el ingreso laboral promedio mensual en lempiras de las mujeres respecto al de los hombres, según área de residencia. 2001, 2005, 2010

\begin{tabular}{|c|l|c|c|c|}
\hline $\begin{array}{c}\text { Área de } \\
\text { Residencia }\end{array}$ & Sexo & 2001 & 2005 & 2010 \\
\hline \multirow{2}{*}{ Urbana } & Hombre & $3,854.10$ & $4,462.50$ & $7,170.90$ \\
\cline { 2 - 5 } & Mujer & $2,624.70$ & $3,279.80$ & $5,668.30$ \\
\hline \multirow{2}{*}{ Rural } & Hombre & 1,38490 & $1,752.10$ & $2,903.60$ \\
\cline { 2 - 5 } & Mujer & $1,231.40$ & $2,000.10$ & $2,374.80$ \\
\hline
\end{tabular}

Fuente: Elaboración propia en base a datos de la EPHPM, 2001, 2005, 2010

\subsection{Ingresos monetarios según sexo y dominio}

Se consideran en esta categoría los ingresos percibidos por los ocupados en concepto de sueldo, salario, jornal, beneficio o ganancia, tanto en la ocupación principal $Y$ secundaria, como los ingresos provenientes de pensiones, subsidios, alquileres, bonos, ayudas familiares, ayudas particulares, remesas del exterior y otros. Los ingresos monetarios son los obtenidos en moneda nacional o extranjera (INE, EPHPM 2001).

Con relación a los ingresos de hombres y mujeres las cifras demuestran que, en las dos grandes ciudades, en promedio, los hombres son mejor retribuidos que el resto de los grupos en los dominios complementarios. En contraposición las mujeres rurales, reciben menores retribuciones, cuya media, en el período estudiado fue de L.1,957.73. El ingreso mensual de los hombres en el dominio del Distrito Central osciló entre L. 4,398.5 y L. 9,048.2 en promedio significó L. 6,254.50; San Pedro Sula presentó cifras similares los salarios se movieron en la banda de L. 4,780.9 y L. 8179.8, lo cual significó un salario intermedio de L. 6,145.47. En todos los dominios las mujeres reciben salarios inferiores a los hombres, con diferencias desde L. 259.20, en el área rural y L.1, 674.93, en San Pedro Sula (Cuadro $N^{\circ} 5$ ).

Cuadro $N^{\circ} 5$ Ingreso mensual de hombres y mujeres por dominio en ocupación principal, en lempiras 2001,2005

$$
\text { y } 2010
$$

\begin{tabular}{|l|c|c|c|c|c|c|c|c|c|}
\hline Dominio & \multicolumn{3}{|c|}{2001} & \multicolumn{3}{c|}{2005} & \multicolumn{3}{c|}{2010} \\
\hline & Total & Hombre & Mujer & Total & Hombre & Mujer & Total & Hombre & Mujer \\
\hline Nacional & 2709.60 & 2947.40 & 2334.80 & 2960.00 & 3029.80 & 2840.20 & 4706.40 & 4750.70 & 4628.90 \\
\hline $\begin{array}{l}\text { Distrtito } \\
\text { Central }\end{array}$ & 3804.10 & 4398.50 & 3102.60 & 4888.60 & 5316.80 & 4389.00 & 8213.40 & 9048.20 & 7257.00 \\
\hline $\begin{array}{l}\text { San } \\
\text { Pedro } \\
\text { Sula }\end{array}$ & 4081.50 & 4780.90 & 3129.30 & 4819.60 & 5475.70 & 3999.50 & 7321.60 & 8179.80 & 6282.80 \\
\hline $\begin{array}{l}\text { Resto } \\
\text { Urbano }\end{array}$ & 2931.80 & 3265.30 & 2480.60 & 3248.60 & 3623.30 & 2755.40 & 5507.40 & 5761.80 & 5150.60 \\
\hline Rural & 1729.00 & 1857.10 & 1462.60 & 1841.20 & 1854.80 & 1807.40 & 2841.10 & 2938.90 & 2603.20 \\
\hline
\end{tabular}

Fuente: Elaboración propia en base a datos de la EPHPM, 2001, 2005, 2010

\section{6. Índice de disimilitud}

Los desajustes en la distribución de las mujeres y los hombres en las diversas ocupaciones laborales pueden verificarse por un índice de segregación. El método ampliamente empleado, en investigaciones, es el creado en 1955 por Duncan y Duncan. Con base en los datos censales, o encuestas de hogares, este método concede la posibilidad de verificar la clasificación de la fuerza laboral para demostrar el grado en el que difiere la participación relativa de los hombres y las mujeres.

Para identificar los índices de segregación de género por sector, en el ámbito centroamericano, durante el período comprendido entre los años 1997 - 2006 el Banco Mundial, en uno de sus estudios aplicó el índice de disimilitud de Duncan. Los datos revelaron que el nivel de segregación era relativamente alto los valores oscilaron entre $0.38 \mathrm{y}$ 0.55 . Esta fuente de asistencia financiera y técnica para los países en desarrollo de todo el mundo asevera que el mercado laboral en la región centroamericana en la década 1997- 2006, presenta un patrón de comportamiento de concentración y segregación de hombres y mujeres en todos los sectores e industrias.

En el caso de Honduras, esa tendencia no ha cambiado durante el decenio bajo revisión. Al aplicar el índice de Duncan por categoría de ocupación en el área rural para 2001 los datos presentan $64.7 \% 2005$ y en 2010 son un tanto similares, $53.9 \%$ y $54.8 \%$ respectivamente. En el área urbana el nivel de segregación más baja se presenta en 2001 con una disimilitud de $36.6 \%$, tanto el 2005 , como en el 2010 las cifras se incrementan 39.8 y $39.7 \%$ (Cuadros $N^{\circ} 6$ al $\mathrm{N}^{\circ} 8$ ).

A la segregación sexual en el trabajo se le asignan múltiples orígenes, Anker y Melkas (2003) estiman que las causas básicas son: sociales, económicas, culturales e históricas. Éstas determinan el alcance y los patrones de segregación en el trabajo en todo el mundo. Asimismo, destacan que los factores que influyen en este fenómeno se refieren a las normas sociales y percepciones estereotipadas respecto a los hombres y las mujeres, la vida familiar, las responsabilidades familiares, la educación y la formación profesional, y la estructura del mercado laboral, entre otras.

El imaginario social consigna a las mujeres los trabajos relativos al cuidado del hogar como una actividad exclusivamente femenina. Por otra parte, a los hombres se les concibe en ocupaciones de toma de decisiones y tareas que se requiere mayor fuerza física.

Con base en los datos del INE 2001, en el área urbana, a través del índice de Duncan, se comprueba que las categorías ocupacionales que más contribuyeron a 
identificar la concentración y segregación fueron la de los servicios cuya diferencia de ocupación por sexo representa el $13.6 \%$. Las mujeres están claramente segregadas en trabajos asociados a la actividad doméstica, al comercio y las ventas; incorporadas con $21.9 \%$ y $27.1 \%$ respectivamente. En contraposición los hombres en las mismas categorías representan el $8.5 \%$ y $17.4 \%$. En posición contraria se presenta la categoría agrícola, donde se observó una diferencia en la participación de las mujeres (9.6\%); son los hombres que se encuentran altamente concentrados en esos quehaceres y en los trabajos de la industria textil, en oficios de la albañilería, y la mecánica, la diferencia por sexo constituye el $15.9 \%$ (Cuadro $\mathrm{N}^{\circ} 6 \mathrm{y} \mathrm{N}^{\circ} 7$ ).

Los datos otorgan la posibilidad de asegurar que las categorías ocupacionales de agricultores, ganaderos y trabajo agropecuario, también los trabajos en la industria, textil, albañilería y mecánica, de seguir el comportamiento descrito, estarían próximas a declararse un caso de segregación total o disimilitud absoluta. En estas ocupaciones prevalece la participación de los hombres, claramente la fuerza laboral fluye a la masculinización de las labores del sector primario, así como las obras de albañilería y mecánica.

La magnitud de la participación de ambos grupos en la categoría ocupacional de directores, gerentes y administración general, no son iguales. Sin embargo, tanto hombres como mujeres representan valores cercanos en el conjunto de la fuerza laboral de esa categoría; en consecuencia la disimilitud es relativa.

Las luchas de las organizaciones feministas y el marco jurídico; podrían ser algunas de las variables influyentes para la apertura de espacios laborales gerenciales y administrativos para las mujeres.

Siguiendo el método de Duncan, aplicado a los datos estadísticos oficiales, el mercado laboral hondureño durante el decenio en estudio no puede declararse con índices extremos. No existe una similitud absoluta, la cual significaría cero segregación o integración completa, tampoco una disimilitud dominante que representa el $100 \%$, total segregación. En su conjunto las categorías ocupacionales presentan diferencias porcentuales por sexo, en la distribución de la fuerza laboral, no obstante, la participación de ambos grupos se refleja en todas las categorías ocupacionales.
Cuadro No6 Índice de disimilitud de Duncan PEA ocupada según grupo ocupacional y dominio 2001

\begin{tabular}{|c|c|c|c|c|}
\hline Ocupación principal Área Urbana 2001 & Empleados & H & M & Diferencia \\
\hline Profesionales, técnicos y PEOA & 150,539 & 11.1 & 15.7 & 4.6 \\
\hline Directores gerentes y administración general & 60,823 & 5.9 & 4.5 & 1.4 \\
\hline Empleados de oficina & 79,162 & 4.8 & 9.5 & 4.6 \\
\hline Comerciantes y vendedores & 249,360 & 17.4 & 27.1 & 9.7 \\
\hline 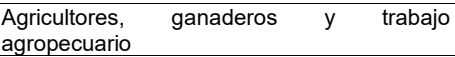 & 66,438 & 10.1 & 0.5 & 9.6 \\
\hline Conductores de transporte & 47,021 & 7.4 & 0.1 & 7.3 \\
\hline $\begin{array}{l}\text { Trabajos industria, textil, albañilería, } \\
\text { mecánica. }\end{array}$ & 219,658 & 26.3 & 10.3 & 15.9 \\
\hline Trabajos área gráfica, química, alimentos. & 78,615 & 5.0 & 9.1 & 4.1 \\
\hline Operador de carga y almacenaje & 29,752 & 3.7 & 1.3 & 2.4 \\
\hline Ocupación de los servicios & 164,593 & 8.3 & 21.9 & 13.6 \\
\hline \multicolumn{4}{|l|}{ Índice de Duncan urbano } & 36.6 \\
\hline \multicolumn{5}{|l|}{ Ocupación Principal -Área Rural, 2001} \\
\hline Profesionales, técnicos y PEOA & 43,521 & 1.9 & 8.4 & 6.4 \\
\hline Directores gerentes y administración general. & 14,021 & 1.0 & 1.7 & 0.7 \\
\hline Empleados de oficina & 10,609 & 0.7 & 1.5 & 0.8 \\
\hline Comerciantes y Vendedores & 138,477 & 4.6 & 30.9 & 26.3 \\
\hline $\begin{array}{llll}\begin{array}{l}\text { Agricultores, } \\
\text { agropecuario }\end{array} & \text { ganaderos } & \text { y } & \text { trabajo } \\
\end{array}$ & 678,250 & 73.6 & 11.7 & 61.9 \\
\hline Conductores de transporte & 17,096 & 2.0 & 8.4 & 6.4 \\
\hline Trabajo industria textil, albañilería, mecánica. & 103,185 & 8.8 & 16.8 & 8.0 \\
\hline Trabajo área gráfica, química, alimentos. & 75,573 & 2.6 & 2.1 & 0.4 \\
\hline Operador de carga y almacenaje & 27,780 & 2.4 & 18.6 & 16.2 \\
\hline Ocupación de los servicios & 79,923 & 2.4 & - & 2.4 \\
\hline \multicolumn{4}{|l|}{ Índice de Duncan rural } & 64.7 \\
\hline
\end{tabular}

Fuente: Elaboración propia en base a datos de la EPHPM, 2001

Cuadro $N^{07}$ Índice de disimilitud de Duncan PEA ocupada según grupo ocupacional y dominio 2005

\begin{tabular}{|c|c|c|c|c|}
\hline Ocupación Principal - Área Urbana, 2005 & Empleados & $\mathbf{H}$ & M & Diferencia \\
\hline Profesionales, técnicos y PEOA & 179,333 & 11.8 & 16.3 & 4.5 \\
\hline Directores, gerentes y administración general & 58,903 & 5.1 & 3.9 & 1.2 \\
\hline Empleados de Oficina & 54,069 & 2.5 & 6.2 & 3.7 \\
\hline Comerciantes y Vendedores & 271,375 & 16.5 & 26.3 & 9.8 \\
\hline $\begin{array}{l}\text { Agricultores, Ganaderos y Trabajo } \\
\text { Agropecuaria }\end{array}$ & 92,666 & 12.0 & 1.4 & 10.6 \\
\hline Conductores de Transporte & 46,208 & 6.5 & 0.1 & 6.4 \\
\hline Trabajos Industria Textil, Albañilería, Mecánica & 252,935 & 28.3 & 9.1 & 19.2 \\
\hline Trabajos área Gráfica, Quim., Alimentos. & 80,110 & 3.3 & 9.6 & 6.3 \\
\hline Operador de Carga y Almacenaje & 33,278 & 3.4 & 1.6 & 1.8 \\
\hline Ocupación de los Servicios & 209,862 & 8.8 & 25.0 & 16.2 \\
\hline \multicolumn{4}{|l|}{ Índice de Duncan urbano } & 39.8 \\
\hline \multicolumn{5}{|l|}{ Ocupación Principal -Área Rural, 2005} \\
\hline Profesionales, Técnicos y PEOA & 39,756 & 1.7 & 6.8 & 5.1 \\
\hline Directores Gerentes y Administración General & 19,647 & 1.4 & 1.6 & 0.2 \\
\hline Empleados de Oficina & 8,090 & 0.4 & 1.2 & 0.7 \\
\hline Comerciantes y Vendedores & 136,604 & 4.6 & 27.0 & 22.4 \\
\hline $\begin{array}{l}\text { Agricultores, Ganaderos y Trabajo } \\
\text { Agropecuaria }\end{array}$ & 778,017 & 71.2 & 20.6 & 50.6 \\
\hline Conductores de Transporte & 19,185 & 1.9 & 0.1 & 1.8 \\
\hline Trabajos Industria, Textil, Albañilería, Mecánica. & 146,543 & 11.3 & 10.2 & 1.0 \\
\hline Trabajo Área Gráfica, Química, Alimentos. & 67,724 & 2.6 & 12.4 & 9.8 \\
\hline Operador de Carga y Almacenaje & 19,185 & 1.5 & 1.3 & 0.2 \\
\hline Ocupación de los Servicios & 88,758 & 2.6 & 18.6 & 16.0 \\
\hline \multicolumn{4}{|l|}{ Índice de Duncan rural } & 53.9 \\
\hline
\end{tabular}

Fuente: Elaboración propia en base a datos de la EPHPM, 2005 
Cuadro N8 Índice de disimilitud de Duncan PEA ocupada según grupo ocupacional y dominio 2010

\begin{tabular}{|l|c|c|c|c|}
\hline \multicolumn{1}{|c|}{ Ocupación Principal- Área Urbana, 2010} & Empleados & $\mathrm{H}$ & $\mathrm{M}$ & Diferencia \\
\hline Profesionales, técnicos y PEOA & 217,029 & 12.0 & 15.7 & 3.7 \\
\hline Directores, gerentes y administración general & 84,559 & 6.1 & 4.5 & 1.6 \\
\hline Empleados de oficina & 74,822 & 3.3 & 9.5 & 6.2 \\
\hline Comerciantes y vendedores & 296,579 & 15.6 & 7.1 & 11.5 \\
\hline Agricultores, ganaderos y trabajo agropecuario & 107,917 & 12.1 & 0.5 & 11.6 \\
\hline Conductores de transporte & 59,508 & 7.1 & 0.1 & 7.0 \\
\hline Trabajos industria textil, albañilería, mecánica & 258,510 & 26.1 & 10.3 & 15.8 \\
\hline Trabajos área gráfica, química, alimentos & 88,317 & 3.2 & 9.1 & 5.9 \\
\hline Operador de carga y almacenaje & 37,718 & 3.4 & 1.3 & 2.1 \\
\hline Ocupación de los servicios & 233,808 & 7.9 & 21.9 & 14.0 \\
\hline İndice de Duncan Urbano & & & & 3 \\
\hline \multicolumn{1}{|c|}{ Ocupación Principal -Área Rural, 2010 } & & & & \\
\hline Profesionales, técnicos y PEOA & 49,136 & 1.8 & 5.5 & 3.7 \\
\hline Directores, gerentes y administración general & 23,227 & 1.3 & 1.4 & 0.1 \\
\hline Empleados de oficina & 10,031 & 0.4 & 1.1 & 0.8 \\
\hline Comerciantes y vendedores & 201,157 & 4.1 & 30.5 & 26.3 \\
\hline Agricultores, ganaderos y trabajo agropecuario & 1087,616 & 75.4 & 26.1 & 49.3 \\
\hline Conductores de transporte & 26,016 & 2.0 & 0.1 & 1.9 \\
\hline Trabajos industria textil, albañilería, mecánica & 145,048 & 9.1 & 5.9 & 3.2 \\
\hline Trabajos área gráfica, química, alimentos & 67,052 & 1.5 & 10.0 & 8.5 \\
\hline Operador de carga y almacenaje & 19,740 & 1.1 & 1.2 & 0.1 \\
\hline Ocupación de los servicios & 114,901 & 2.1 & 18.0 & 15.8 \\
\hline Índice de Duncan Rural & & & 54.8 \\
\hline
\end{tabular}

Fuente: Elaboración propia en base a datos de la EPHPM, 2010

\section{Conclusiones}

A la luz de los datos estadísticos, en el período estudiado, la ocupación desagregada por sexo identificada en cada una de la naturaleza ocupacional, remarcan los patrones culturales y las características históricas relativas a la asignación de roles diferenciados para hombres y mujeres convirtiéndose en un elemento determinante para la segregación laboral.

Se identifica claramente la masculinización de las ocupaciones de agricultores, ganadería y trabajo agropecuario, así como los trabajos en la industria, textil, en los oficios de albañilería y mecánica. De mantenerse esa tendencia este comportamiento del mercado laboral estaría próximo a declararse un caso de segregación total o disimilitud absoluta.

El dominio en apariencia podría ser un factor que determina la segregación laboral, en virtud de que el índice de disimilitud marca una diferencia muy superior de ocupación por sexo entre el ámbito urbano y rural. No obstante, tal disimilitud, se debe a que la ocupación de mayor importancia en al ámbito rural son las tareas que pertenecen el sector primario de la economía.

Los resultados demuestran tanto la importancia cuantitativa del fenómeno como su estrecha relación con los estereotipos de género. De las diez ocupaciones categorizadas por el INE, en cinco de ellas la representación masculina supera a sus homologas. Son los varones los que marcan la tendencia nacional de las ocupaciones. La alta presencia de hombres se distingue en posiciones directivas y gerenciales, la agricultura, conducción de transporte, operación de carga y almacenaje, trabajos en la industria textil y tareas de albañilería, mecánica y otros.

El salario es un factor influyente en la segregación laboral. Los y las trabajadoras reciben mensualmente pagos superiores cuando laboran en el área urbana. Los varones reciben remuneraciones más altas que las mujeres tanto en el ámbito urbano como en el espacio rural. Con relación al promedio del ingreso laboral entre hombres y mujeres en el área urbana se mantiene la brecha salarial. Las diferencias salariales alcanzan hasta 20 puntos porcentuales.

\section{Bibliografía}

- Anker.R, Melkas.H, Korten. A (2003). Gender-based occupational segregation in the 1990's. Disponible en: http://natlex.ilo.ch/wcmsp5/groups/public/---ed_norm/--declaration/documents/publication/wcms_decl_wp_18_ en.pdf. Recuperado 5/01/2015

- Cálix M.(2017) Segregación Ocupacional Femenina En El Mercado Laboral De Honduras 2001, 2005, 2010.

- De Oliveira Soruce.O. (2007) Reflexiones acerca de las desigualdades sociales y el género. Estudios Sociológicos, Vol. 25, No. 75 (Sep. - Dec., 2007), pp. 805-812 Published by: El Colegio de México Stable URL: Disponible en: http:// www.jstor.org/stable/40421109 . Recuperado 25-01-2018 19:22 UTC

- Instituto Nacional de Estadísticas (INE). Disponible en : http://www.ine.gob.hn/index.php/menu-descargas Recuperado 2/12/2014

- OIT (2003) La Hora De La Igualdad En El Trabajo Disponible en: http://www.ilo.org/wcmsp5/groups/public/--dgreports/---dcomm/---publ/documents/publication/wcms_ publ_9223128714_sp.pdf Recuperado 28-12-2017 